\title{
Relação entre a gordura corporal e indicadores antropométricos em adultos frequentadores de academia
}

\author{
Relationship between percentage of body fat and anthropometric \\ indicators in individuals attending a gym
}

\author{
T. Grossl, L.R. Augustemak de Lima, F.C. Karasiak
}

\begin{abstract}
RESUMO
O objetivo do estudo foi verificar a relação entre o percentual de gordura corporal (\% GC) e indicadores antropométricos em frequentadores de academia. A amostra foi composta por 438 alunos frequentadores de academia, sendo 195 homens e 243 mulheres, amplitude de 18 a 50 anos de idade. O \% GC foi estimado pelo método das dobras cutâneas. Os seguintes indicadores antropométricos foram avaliados: circunferência da cintura, circunferência do abdômen (CA), razão cintura quadril, índice de massa corporal (IMC) e razão cintura estatura. Utilizou-se da correlação linear de Pearson e análise de regressão linear simples para verificar a relação entre as variáveis. No sexo feminino, o IMC apresentou-se como o indicador mais fortemente correlacionado com o \% GC $(r=.73)$, enquanto que para o sexo masculino, a CA demonstrou maior correlação com o \% GC $(r=.73)$. Foram observadas correlações significantes entre todos os indicadores antropométricos analisados e \% GC, com variações em sua magnitude.

Palavras-chave: composição corporal, índice de massa corporal, circunferência abdominal
\end{abstract}

ABSTRACT

The aim of this study was to investigate the relationship between percentage of body fat (\% BF) and anthropometric indicators in individuals attending a gym. Four hundred and thirty eight individuals, 195 men and 243 women, from 18 to 50 years of age took part in this study. The \% BF was estimated by the skinfold method. The following anthropometric indicators were assessed: waist circumference, abdomen circumference (AC), waist-to-hip ratio, body mass index (BMI) and waist-height ratio. Linear Pearson correlation and simple linear regression analysis were used to investigate the relationship between variables. For women, BMI strongly correlated with \% BF $(r=.73)$, whereas for males, AC showed high correlation with $\% \mathrm{BF}(r=.73)$. With varying degrees of magnitude, there were significant correlations between all of the anthropometric indicators analyzed and \% BF.

Keywords: body composition, body mass index, abdomen circumference

Submetido: 13.09.2009 | Aceite: 30.03.2010

Talita Grossl e Fábio Colussi Karasiak. Mestrando do Programa de Pós Graduação em Educação Física da Universidade Federal de Santa Catarina (UFSC), Laboratório de Esforço Físico (LAEF), Florianópolis (SC) - Brasil.

Luiz Rodrigo Augustemak de Lima. Mestrando do Programa de Pós Graduação em Educação Física da Universidade Federal de Santa Catarina (UFSC), Núcleo de Pesquisa em Cineantropometria e Desempenho Humano (NUCIDH), Florianópolis (SC) - Brasil.

Endereço para correspondência: Talita Grossl, Universidade Federal de Santa Catarina, Centro de Desportos Laboratório de Esforço Físico, Campus Universitário - Trindade, CEP: 88040-900, Florianópolis, SC., Brasil.

E-mail: talitagrossl@gmail.com 
A literatura tem apontado que a concentração de gordura na região abdominal, independentemente da gordura corporal total, é fator determinante de múltiplos distúrbios cardiovasculares e metabólicos (Goodpaster et al. 2005; Rexrode et al., 1998; Silva, Barbosa, Oliveira, \& Guedes, 2006). O aumento excessivo da gordura corporal está fortemente associado com o risco de morte (Wannamethee, Shaper, Lennon, \& Whincup, 2007), representando assim, um dos maiores problemas atuais de saúde pública (Cavalcanti, Carvalho, \& De Barros, 2009).

A absortometria radiológica de dupla energia (DXA) é um método bem estabelecido no meio científico, com acurácia e reprodutibilidade válida na estimativa da gordura corporal, no entanto, é uma técnica que requer elevado custo operacional e técnicos especializados (Ellis, 2000). Dessa forma, métodos indiretos como a mensuração de dobras cutâneas têm sido utilizados, por meio de equações de regressão para a estimativa da gordura corporal, tendo como vantagens a aplicabilidade em grandes grupos, rapidez, baixo custo operacional e ser um método não invasivo (Carvalho \& Pires Neto, 1999).

Ainda no contexto antropométrico, vários indicadores são propostos na literatura para a avaliação do estado nutricional, bem como o diagnóstico de riscos à saúde por conta de aumentos na gordura corporal. $O$ índice de massa corporal (IMC), desenvolvido por Quetelet em 1871, é um dos procedimentos mais usados para avaliação do excesso de peso e obesidade em estudos epidemiológicos (NHLBI, 1998; Rech, Petroski, Silva, \& Silva, 2006).

Para a Organização Mundial da Saúde (OMS), além da massa corporal e da estatura, devem ser medidos a circunferência da cintura (CC) e circunferência do quadril (CQ), pois o aumento da deposição de gordura abdominal na população pode fornecer um indicador sensível (WHO, 1995). Entretanto, estudos apontam para perda da sensibilidade quando utilizado a razão cintura-quadril (RCQ) como indicador de risco, pois o quadril inclui a gordura subcutânea pélvica, a massa muscular e o tamanho do osso pélvico horizontal (Page et al., 2009). Uma alternativa proposta, ainda utilizando a CC, é a razão cintura-estatura (RCE), onde ocorre um ajuste da CC pela estatura (Flegal et al., 2009). A circunferência abdominal (CA) também tem sido utilizada como um indicador da distribuição central da gordura corporal (Alvarez, Vieira, Sichieri, \& Veiga, 2008). Nesse sentido, os indicadores antropométricos têm como objetivo refletir o excesso de gordura corporal, esse fato se torna importante pela praticidade na utilização desses indicadores por profissionais na área da saúde.

Os profissionais envolvidos em programas interdisciplinares para redução/controle da gordura corporal necessitam de ferramentas válidas para prescrever exercícios e monitorar a efetividade do programa em grandes grupos de praticantes, sobretudo quando o objetivo é a modificação na quantidade de gordura corporal (Costa, Guiselini, \& Fisberg, 2007). Assim pode-se hipotetizar que os indicadores antropométricos (IMC, CC, RCQ, RCE e CA) possuem relação com o percentual de gordura corporal (\% GC) em frequentadores de academia de ambos os sexos.

Dessa forma, os objetivos do presente estudo foram: 1) verificar a utilidade dos indicadores antropométricos (IMC, CC, RCQ, RCE e CA) em frequentadores de academia de ambos os sexos; 2) analisar e comparar as diferenças dos indicadores entre as faixas etárias e sexos; e, 3) verificar a magnitude da relação entre os indicadores antropométricos com o \%GC.

\section{MÉTODO}

\section{Amostra}

Participaram do estudo 438 alunos praticantes de exercícios físicos de uma academia de ginástica de Florianópolis/SC/Brasil, sendo 195 homens e 243 mulheres, com idade média de $31.3 \pm 8.1$ anos e $32.2 \pm 8.6$ anos, 
respectivamente, com amplitude de 18 a 50 anos de idade. Foram selecionados por procedimento não-probabilístico, do tipo intencional, onde os alunos que realizavam exercícios resistidos por no mínimo um mês passaram por uma rotina de avaliações de aptidão física, incluindo a realização de medidas antropométricas. Esta pesquisa seguiu os princípios éticos de respeito à autonomia das pessoas, apontados pela Resolução $\mathrm{n}^{\circ}$ 196/1996, do Conselho Nacional de Saúde. Todos os sujeitos que participaram do estudo assinaram um termo de consentimento livre e esclarecido.

\section{Procedimentos}

Foram mensuradas as variáveis de massa corporal e estatura por meio de uma balança eletrônica WELMY, modelo R-110 (precisão de $.1 \mathrm{~kg}$ ) e estadiômetro portátil SANNY, EUA (precisão de .1 cm), respectivamente, atendendo às padronizações sugeridas por Gordon, Chumlea e Roche (1991). As medidas de peso e estatura foram utilizadas para o cálculo do IMC, onde o peso $(\mathrm{kg})$ é dividido pelo quadrado da estatura (m), utilizando-se os pontos de corte propostos pela WHO (1995).

A medida da CC foi realizada utilizando o procedimento descrito por Callaway et al. (1991), onde a fita inelástica é colocada horizontalmente, no seu menor perímetro. As medidas foram realizadas com a fita sobre a pele, todavia sem compressão dos tecidos. Foi utilizada uma fita métrica flexível com precisão de $1 \mathrm{~mm}$. A RCE foi calculada por meio da razão da $C C(\mathrm{~cm})$ pela estatura $(\mathrm{cm})$.

A CQ foi mensurada com uma fita métrica, colocada horizontalmente em volta do quadril na parte mais saliente dos glúteos (Callaway et al., 1991). A RCQ foi obtida pela divisão da CC $(\mathrm{cm})$ pela CQ $(\mathrm{cm})$. A CA foi identificada em seu maior perímetro, não sendo necessariamente localizada sobre a cicatriz umbilical (Callaway et al., 1991). A fita foi colocada em torno do abdômen do avaliado, de trás para frente, mantendo-a no plano horizontal e após uma expiração normal.
Para a medida critério, foram mensuradas as dobras cutâneas com a utilização de um plicômetro SANNY, modelo AD-1010 (precisão de $1 \mathrm{~mm}$ ). Todas as dobras cutâneas foram realizadas no hemicorpo direito do sujeito, seguindo o padrão descrito por Harisson et al. (1991). Mensuraram-se três medições em todas as medidas antropométricas, adotando-se a média como valor final, assim como foram realizadas por um mesmo avaliador. A estimativa da densidade corporal foi obtida pelo modelo de regressão proposto por Jackson e Pollock (1978) para homens, onde são mensuradas três dobras cutâneas (peitoral, abdominal e coxa média). Para as mulheres foram mensuradas as dobras cutâneas das regiões tricipital, supra-ilíaca e coxa média (Jackson, Pollock, \& Ward, 1980). O cálculo do \% GC foi realizado pela conversão da densidade corporal utilizando a equação de Siri (1961).

\section{Análise estatística}

Empregou-se a análise descritiva (média, desvio-padrão e amplitudes) para apresentação inicial dos resultados. Verificou-se a distribuição normal dos dados por meio da análise gráfica dos histogramas. A comparação entre os sexos foi testada por meio do teste $t$ de Student para amostras independentes, ainda, para testar a diferença entre as faixas etárias, utilizou-se a ANOVA one-way, seguido do post-hoc de Tukey. Para verificar a relação dos indicadores antropométricos com o \% GC, utilizou-se a correlação linear de Pearson e a análise de regressão linear simples, respectivamente. Em todas as análises foi utilizado o pacote estatístico Statistical Package for the Social Sciences (SPSS), versão 15.0 para Windows, estabelecendo um nível de significância de $5 \%$.

\section{RESULTADOS}

Com o objetivo de visualizar as características da amostra avaliada, o quadro 1 apresenta os valores médios e desvios padrão dos participantes do estudo, estratificada por sexo 
e faixas etárias. Em todas as variáveis analisadas, houve diferenças significativas entre os sexos $(p<.05)$.

De acordo com a classificação do IMC proposta pela WHO (1995), verificou-se uma frequência relativa de $42.1 \%$ dos sujeitos, do sexo masculino, sendo identificados como eutróficos, $47.1 \%$ apresentaram sobrepeso e $10.8 \%$ obesidade. Em contrapartida, para o sexo feminino, observou-se que $84.8 \%$ foram classificadas como eutróficas, $11.9 \%$ apresentaram sobrepeso e $3.3 \%$ obesidade.

Quadro 1

Valores médios e desvios padrão das características antropométricas dos participantes do estudo

\begin{tabular}{|c|c|c|c|c|}
\hline \multirow[b]{2}{*}{ Faixa Etária (anos) } & \multicolumn{4}{|c|}{ Masculino $^{\$ S}$} \\
\hline & $\begin{array}{c}18 \text { a } 29 \\
(n=92)\end{array}$ & $\begin{array}{c}30 \text { a } 39 \\
(n=70)\end{array}$ & $\begin{array}{c}40 \text { a } 50 \\
(n=33)\end{array}$ & $\begin{array}{c}\text { Total } \\
(n=195)\end{array}$ \\
\hline Massa corporal (kg) & $82.4 \pm 11.7$ & $84.5 \pm 12.3$ & $79.2 \pm 10.3$ & $82.6 \pm 11.8$ \\
\hline Estatura $(\mathrm{cm})$ & $179.0 \pm 5.9^{* *}$ & $177.8 \pm 7.1$ & $175.2 \pm 6.1$ & $177.9 \pm 6.5$ \\
\hline$\%$ GC $(\%)$ & $14.8 \pm 5.2^{*}$ & $18.7 \pm 4.7$ & $18.9 \pm 4.4$ & $16.9 \pm 5.3$ \\
\hline \multirow[b]{2}{*}{ Faixa Etária (anos) } & \multicolumn{4}{|c|}{ Feminino } \\
\hline & $\begin{array}{c}18 \text { a } 29 \\
(n=104)\end{array}$ & $\begin{array}{c}30 \text { a } 39 \\
(n=84)\end{array}$ & $\begin{array}{l}40 \text { a } 50 \\
(n=55)\end{array}$ & $\begin{array}{c}\text { Total } \\
(n=243)\end{array}$ \\
\hline Massa corporal $(\mathrm{kg})$ & $58.6 \pm 8.4^{* *}$ & $59.9 \pm 8.5$ & $62.9 \pm 10.3$ & $60.0 \pm 9.0$ \\
\hline Estatura $(\mathrm{cm})$ & $164.6 \pm 6.4$ & $164.1 \pm 6.3$ & $163.0 \pm 6.8$ & $164.1 \pm 6.5$ \\
\hline$\%$ GC $(\%)$ & $24.6 \pm 5.4$ & $25.5 \pm 5.6$ & $28.2 \pm 4.9^{\S}$ & $24.7 \pm 6.6$ \\
\hline
\end{tabular}

${ }^{*} p<.05$ em relação às faixas etárias 30 a 39 anos e 40 a 50 anos; ${ }^{* *} p<.05$ em relação à faixa etária 40 a 50 anos; ${ }^{5} p<.05$ em relação às faixas etárias 18 a 29 anos e 30 a 39 anos; ${ }^{55} p<.05$ em relação ao sexo feminino, nas variáveis de massa corporal, estatura e \% GC

O quadro 2 mostra os valores médios e desvios padrão dos indicadores antropométricos. Novamente foram encontradas diferenças significativas entre os sexos em todos indicadores antropométricos avaliados na pesquisa $(p<.05)$.

A faixa etária de 40 a 50 anos apresentou diferença significante $(p<.05)$ para todos os indicadores antropométricos ao serem comparadas com as faixas etárias mais jovens, nas mulheres. Os resultados no sexo masculino mostraram que tanto a CC como RCE foi significantemente menor $(p<.05)$ para o grupo mais jovem comparado com as faixas etárias de 30 a 39 anos e 40 a 50 anos. Já a RCQ apresentou diferença significativa $(p<$ .05) em todos os grupos de homens, divididos por faixas etárias.

O quadro 3 apresenta os valores da correlação linear de Pearson e coeficientes de determinação entre o \% GC com os indicadores antropométricos avaliados. Observa-se que o IMC foi o indicador que teve maior correlação com o \% GC para as mulheres. Entretanto, para esse sexo, estratificado por faixas etárias nota-se uma aproximação muito grande na correlação entre o \% GC com o IMC, CA, CC e RCE. Já para os homens, em todas as faixas etárias, a CA foi indicador antropométrico com mais forte relação com o \% GC. A RCQ foi o indicador com menor relação com o \% GC em ambos os sexos (quadro 3).

A figura 1 apresenta gráficos de dispersão, onde é possível verificar a disposição e tendências na relação entre o IMC e CA com o $\%$ GC, estratificados por sexo. Observa-se maior variabilidade dos respectivos indicadores antropométricos no sexo masculino, quando comparado ao feminino. 
Quadro 2

Valores médios e desvios padrão dos indicadores antropométricos

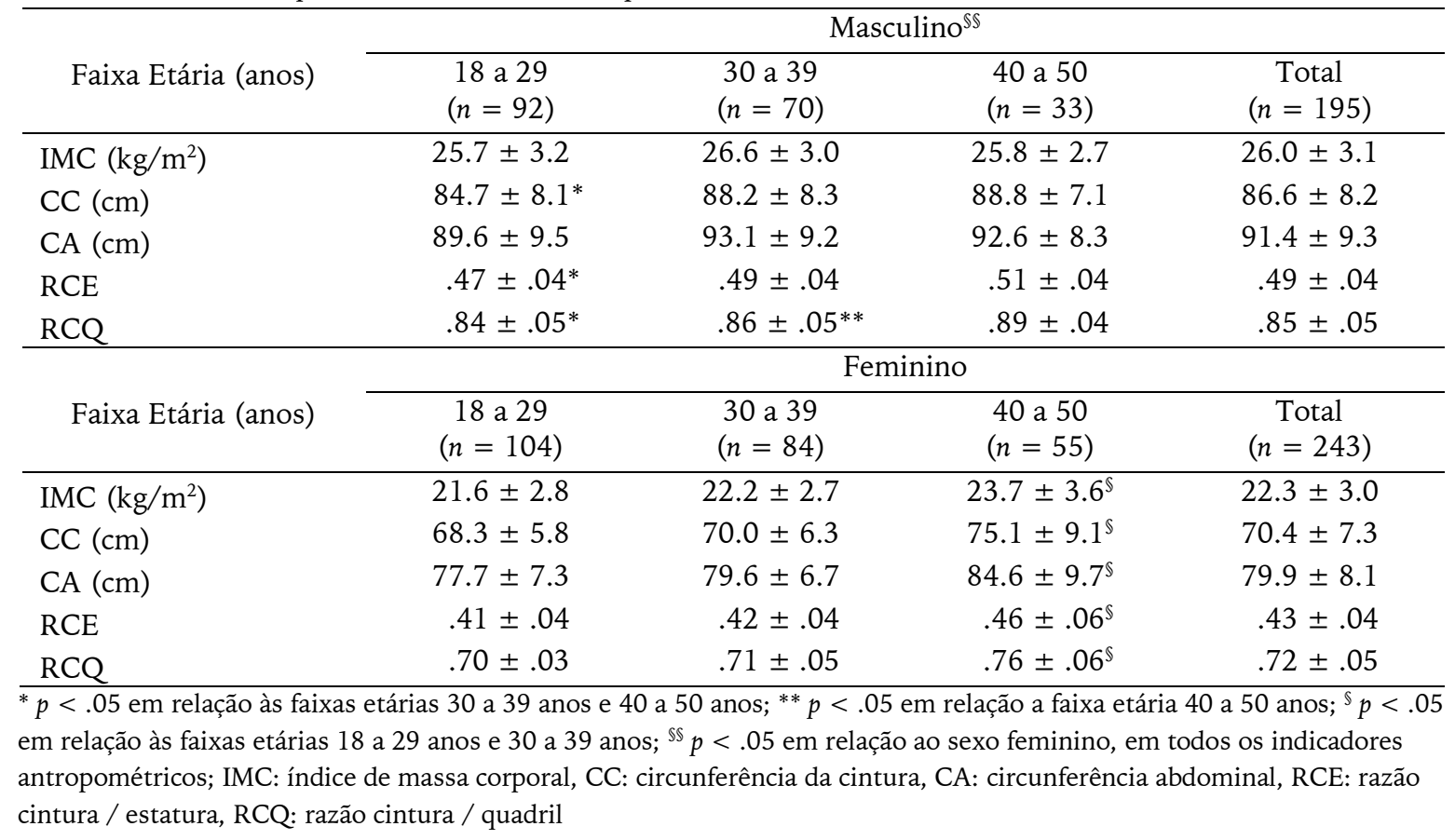

Quadro 3

Coeficientes de correlação linear $(r)$ e coeficientes de determinação $\left(R^{2}\right)$ entre o \% GC com os indicadores antropométricos

\begin{tabular}{|c|c|c|c|c|c|c|c|c|}
\hline \multirow{3}{*}{ Faixa Etária (anos) } & \multicolumn{8}{|c|}{ Masculino } \\
\hline & \multicolumn{2}{|c|}{$\begin{array}{c}18 \text { a } 29 \\
(n=92)\end{array}$} & \multicolumn{2}{|c|}{$\begin{array}{c}30 \text { a } 39 \\
(n=70)\end{array}$} & \multicolumn{2}{|c|}{$\begin{array}{l}40 \text { a } 50 \\
(n=33)\end{array}$} & \multicolumn{2}{|c|}{$\begin{array}{c}\text { Total } \\
(n=195)\end{array}$} \\
\hline & $r$ & $R^{2}$ & $r$ & $R^{2}$ & $r$ & $R^{2}$ & $r$ & $R^{2}$ \\
\hline $\operatorname{IMC}\left(\mathrm{kg} / \mathrm{m}^{2}\right)$ & .49 & .24 & .55 & .30 & .71 & .50 & .54 & .29 \\
\hline RCE & .64 & .41 & .59 & .35 & .70 & .50 & .67 & .45 \\
\hline $\mathrm{RCQ}$ & .39 & .12 & .27 & .07 & .43 & .18 & .43 & .19 \\
\hline $\mathrm{CC}(\mathrm{cm})$ & .66 & .44 & .59 & .34 & .76 & .58 & .67 & .45 \\
\hline \multirow[t]{2}{*}{$\mathrm{CA}(\mathrm{cm})$} & .72 & .51 & .71 & .50 & .81 & .66 & .73 & .53 \\
\hline & \multicolumn{8}{|c|}{ Feminino } \\
\hline \multirow[t]{2}{*}{ Faixa Etária (anos) } & \multicolumn{2}{|c|}{$\begin{array}{c}18 \text { a } 29 \\
(n=104)\end{array}$} & \multicolumn{2}{|c|}{$\begin{array}{c}30 \text { a } 39 \\
(n=84)\end{array}$} & \multicolumn{2}{|c|}{$\begin{array}{l}40 \text { a } 50 \\
(n=55)\end{array}$} & \multicolumn{2}{|c|}{$\begin{array}{c}\text { Total } \\
(n=243)\end{array}$} \\
\hline & $r$ & $R^{2}$ & $r$ & $R^{2}$ & $r$ & $R^{2}$ & $r$ & $R^{2}$ \\
\hline $\operatorname{IMC}\left(\mathrm{kg} / \mathrm{m}^{2}\right)$ & .79 & .62 & .66 & .45 & .68 & .47 & .73 & .53 \\
\hline RCE & .76 & .58 & .59 & .35 & .66 & .43 & .69 & .47 \\
\hline $\mathrm{RCQ}$ & .33 & .12 & $.14^{*}$ & $.02 *$ & .39 & .15 & .34 & .11 \\
\hline $\mathrm{CC}(\mathrm{cm})$ & .77 & .53 & .55 & .30 & .63 & .40 & .67 & .44 \\
\hline $\mathrm{CA}(\mathrm{cm})$ & .72 & .51 & .65 & .42 & .69 & .48 & .70 & .49 \\
\hline
\end{tabular}

${ }^{*}$ Não significante $(p>$.05); IMC: índice de massa corporal, CC: circunferência da cintura, CA: circunferência abdominal, RCE: razão cintura / estatura, RCQ: razão cintura / quadril 
a)

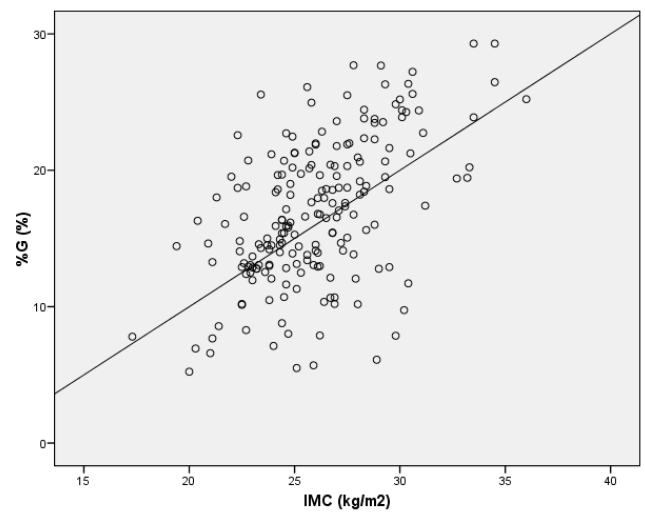

c)

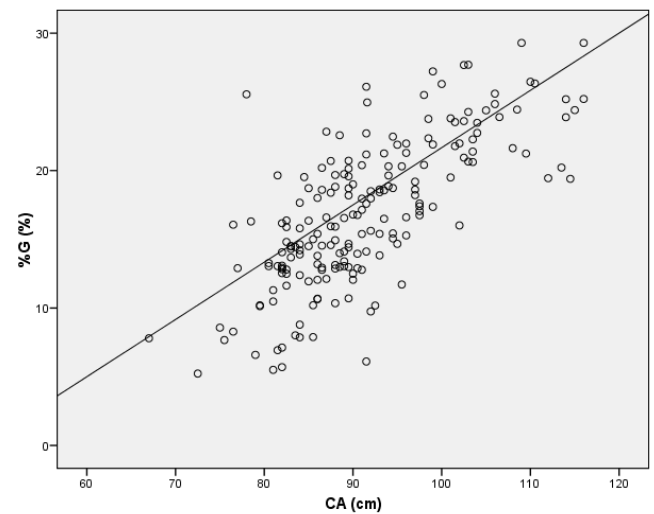

Feminino

b)



d)

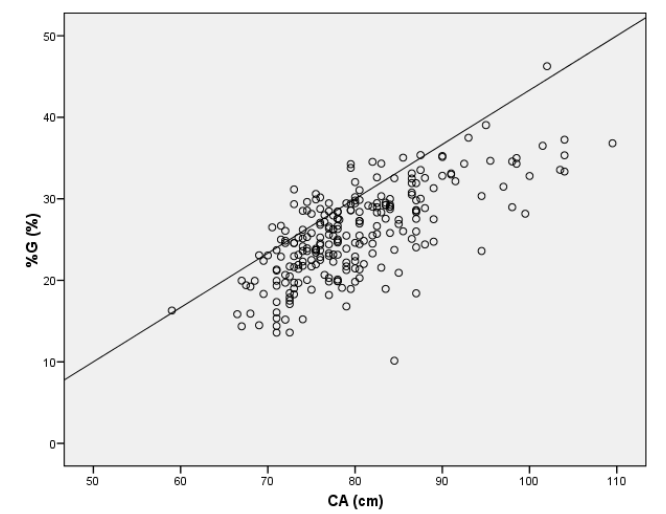

Figura 1. Gráfico de dispersão entre o \% GC e os indicadores antropométricos IMC e CA

As figuras a) e b) são gráficos de dispersão entre as variáveis \% GC e IMC para o sexo masculino e feminino, respectivamente; As figuras: c) e d) são gráficos de dispersão entre as variáveis \% GC e CA para o sexo masculino e feminino, respectivamente

\section{DISCUSSÃO}

O principal objetivo deste estudo foi verificar a relação de diferentes indicadores antropométricos com a gordura corporal relativa em adultos frequentadores de academia de ambos os sexos. $\mathrm{O}$ achado mais importante do presente estudo foi evidenciar que todos os indicadores antropométricos tiveram uma correlação positiva e significante com o \%GC, com ênfase, o IMC e a CA foram os indicadores que apresentaram maior força na relação, em mulheres e homens, respectivamente. Essa análise reforça a possibilidade de utilizar indicadores antropométricos como uma alternativa na avaliação e prescrição de exercícios em grupos de larga escala. A praticidade e o baixo custo na avaliação permitem uma maior amplitude no escopo de monitoramento do estado nutricional da população, auxiliando programas de intervenções a nível epidemiológico.

As medidas antropométricas são frequentemente utilizadas como indicadores da localização de gordura central, em estudos epidemiológicos, que visam identificar o risco para doenças cardiovasculares (Page et al., 2009). No entanto, ainda há discussões sobre a melhor medida para este fim, uma vez que um bom indicador de localização deveria associarse de maneira independente de sexo, idade e adiposidade total, com os marcadores de risco para as doenças cardiovasculares (Alvarez et al., 2008). 
Em estudo recente, Costa et al. (2007), identificaram a relação do \% GC com o IMC em 799 frequentadores de academia com idade entre os 20 e os 50 anos (363 homens e 436 mulheres) e tiveram como resultados uma correlação maior entre as variáveis quando comparado ao atual estudo $\left(R^{2}=.58\right.$ para homens e $R^{2}=.68$ para mulheres). Em adição, os dados também indicaram uma relação maior para as mulheres do que para os homens.

A diferença entre as correlações do \% GC com o IMC entre homens e mulheres $(r=.54$ e $r=.73$, respectivamente) eram esperadas visto que os homens possuem uma maior quantidade de massa corporal magra comparado às mulheres. Nesse caso, com a utilização do IMC existe a possibilidade de diagnosticar falsos-positivos, ou seja, identificar indivíduos como sobrepeso e obesos enquanto eles possuem aumentos na massa corporal magra, indicando um diagnóstico inadequado. No entanto, não foi o objetivo do presente estudo testar a sensibilidade e especificidade dos pontos de corte.

Ao analisar o quadro 3 percebe-se que no sexo masculino, o IMC teve maior correlação com o \% GC na faixa etária de 40 a 50 anos $(r$ $=.71)$ do que as faixas etárias anteriores $(r=$ .49 para 18 a 29 anos e $r=.55$ para 30 a 39 anos). Com o passar dos anos, especialmente dos 40 aos 60 anos de idade, ocorre, gradativamente, um aumento do peso corporal e uma diminuição da estatura, explicada, em grande parte, pela perda de massa óssea, o que influencia diretamente no IMC (Matsudo, Matsudo, \& Barros Neto, 2000). Os autores acrescentam que o processo de envelhecimento inclui um aumento da gordura corporal, diminuição da massa livre de gordura e seus principais componentes (mineral, água, proteína e potássio), diminuição da taxa metabólica de repouso, massa muscular esquelética e massa óssea. Corroborado por um estudo longitudinal, no qual foi identificado aumento na gordura corporal total em 54 homens e 75 mulheres com idade média de $60.4 \pm 7.8$ anos (Hughes et al., 2004).
O IMC explicou, em média, $53 \%$ da variação do \% GC nas mulheres e $29 \%$ nos homens. A correlação do IMC com o \% GC foi mais forte na idade de 18 a 29 anos para as mulheres, explicando $62 \%$ da variação do $\%$ GC, e na faixa etária entre 40 e 50 anos para os homens, explicando $50 \%$ dessa variação, como já demonstrado anteriormente.

Ao analisar a relação do IMC e CC com a distribuição da gordura corporal, Janssen, Heymsfield, Allison, Kotler e Ross (2002) observaram correlações significantes e em magnitudes similares entre o IMC e CC com a gordura total, não abdominal e gordura subcutânea da região abdominal, em mulheres. Enquanto para os homens foram observadas as mesmas correlações com as medidas de gordura corporal, porém o IMC $(r=.78)$ demonstrou maior correlação quando comparado a CC $(r=.68)$, discordando do presente estudo, onde o IMC obteve correlações de menor magnitude, para o sexo masculino. Análises subsequentes da pesquisa supracitada identificaram que a CC, independente do sexo, apresentou correlações mais fortes com a gordura visceral do que o IMC.

A Associação Brasileira para o Estudo da Obesidade e da Síndrome Metabólica (ABESO, 1998) aceita que a simples determinação da CC pode ser suficiente para categorizar, conforme o sexo, o risco de complicações metabólicas. Wang et al. (2003) realizaram comparações entre quatro protocolos utilizados para a mensuração da CC: imediatamente abaixo da última costela, menor circunferência da cintura, ponto médio entre a última costela e a crista ilíaca, e imediatamente acima da crista ilíaca. Os quatro protocolos testados apresentaram alta reprodutibilidade e foram correlacionados significantemente com a gordura corporal total, em ambos os sexos. Entretanto, quando realizada a correlação dos protocolos de CC com o \% GC, somente foram encontradas relações significantes para o sexo feminino. Nesse sentido, os resultados discordaram parcialmente do atual estudo. 
Estas divergências podem, em parte, serem atribuídas às diferenças do método de mensuração da gordura corporal $\mathrm{e}$ às características da amostra (etnia e idade).

$\mathrm{Na}$ adiposidade central, a distribuição do tecido adiposo se dá preferencialmente no nível do tronco com a deposição aumentada na região intra-abdominal (Mancini, 2001). De um modo geral, os estudos utilizam a região da menor circunferência do tronco entre a última costela e a crista ilíaca para a medida da CC (Callaway et al., 1991). Entretanto, alguns autores usam a denominação e os pontos de corte da CC, mas aferem a medida na cicatriz umbilical (Daniels, Khoury, \& Morrison, 2000; Ribeiro, Gimeno, Andreoni, \& Ferreira, 2006). Porém, de acordo com Callaway et al. (1991), a localização desta medida seria para aferição da CA. Esta aparente confusão de padronizações pode gerar incoerências na interpretação de resultados referentes à utilização das medidas antropométricas de acúmulo de gordura central (Alvarez et al., 2008).

Ao analisar os dados da presente investigação, percebe-se que a CA, medida na maior circunferência do abdômen, não sendo necessariamente localizada sobre a cicatriz umbilical (Callaway et al., 1991), foi a mais correlacionada com o \% GC nos homens $(r=$ .73). Ao analisar por faixas etárias, entre os 40 e 50 anos de idade, a CA foi aquela com maior força na correlação com o \% GC para as mulheres $(r=.69)$. Assim, a CA explica $53 \% \mathrm{e}$ $49 \%$ da variação do \% GC em homens e mulheres, respectivamente. Sendo que para o sexo masculino, na faixa etária de 40 a 50 anos, esta variável explicou $66 \%$ da variação do \% GC.

$\mathrm{Na}$ literatura revisada não se encontrou pontos de corte recomendados para discriminar CA com riscos a saúde, entretanto é possível supor que, em função da forte correlação entre CC e CA encontrada no presente estudo $(r=.95$ e $r=.93, p<.001$, para homens e mulheres, respectivamente), os valores dos pontos de corte ideais para os dois parâmetros sejam próximos (Hasselmann,
Faerstein, Werneck, Chor, \& Lopes, 2008). Nessa perspectiva, necessita-se de estudos que realizem pontos de corte para a $\mathrm{CA}$, verificando se os valores ficariam realmente próximos do ponto de corte para a CC, visto que os resultados do atual estudo demonstraram que a medida desta variável foi aquela que melhor se relacionou e explicou as variações do \% GC em homens, principalmente naqueles com faixa etária entre 40 e 50 anos.

Recentemente, a RCE tem sido proposta como uma alternativa no uso de indicadores antropométricos (Flegal et al., 2009). Nesse sentido, estudos têm demonstrado que a RCE está fortemente associada aos diversos fatores de risco coronariano (Ho, Lam, \& Janus, 2003; Lin et al., 2002; Pitanga \& Lessa, 2006). Huang et al. (2002) observaram que existe uma forte associação da RCE com hipertensão arterial, intolerância a glicose, diabetes e dislipidemias, ao avaliarem 38556 sujeitos de ambos os sexos.

Page et al. (2009) realizaram um estudo longitudinal para utilizar a RCE como preditor do risco coronariano entre as mulheres, e encontraram que a $C Q$, IMC e RCQ foram correlacionadas positivamente com a RCE. Os mesmos autores concluíram que a RCE é superior ao IMC para predizer a incidência doenças coronarianas entre mulheres de meiaidade e idosas, sendo comparável com a RCQ e CC.

$\mathrm{Na}$ investigação de Flegal et al. (2009) foram comparados o \% GC (mensurados por meio da DXA) com os indicadores antropométricos (IMC, CC e RCE), em 12901 adultos, estratificados por faixa etária. Os autores observaram que em homens, o \% GC foi mais correlacionado com a CC e RCE quando comparadas ao IMC. Em relação às mulheres, o \% GC foi mais correlacionado com o IMC do que a CC. $\mathrm{Na}$ análise estratificada por faixa etária, o \% GC foi significantemente mais correlacionado com a RCE quando comparada a CC, no grupo mais jovem de ambos os sexos (20 a 39 anos). Estes dados acima corroboram com o atual estudo, onde o 
\% GC, para as mulheres, tendeu a ser levemente mais correlacionada com a RCE $(r=$ .69) quando comparada com a CC $(r=.67)$. No entanto, para os homens, a RCE e CC foram igualmente correlacionadas com o \% GC $(r=.67)$.

A medida da RCQ tem sido utilizada para revelar a distribuição da gordura corporal. Pereira, Sichieri e Marins (1999) acrescentam que não há consenso sobre a definição do que seja uma RCQ elevada. A implicação da realização desta medida está no fato do quadril incluir a gordura subcutânea pélvica, a massa muscular e o tamanho do osso pélvico horizontal (Flegal et al., 2009), dessa forma, causando confusões para a avaliação da gordura corporal. Os resultados da correlação (quadro 3) demonstram que a RCQ foi o indicador com menor relação com o \% GC dentre os indicadores analisados, em ambos os sexos.

Alvarez et al. (2008), ao associarem medidas antropométricas de localização de gordura central com os componentes da síndrome metabólica em adolescentes, verificaram que a RCQ foi a medida que apresentou a menor associação, não tendo efeito significativo para nenhuma das variáveis investigadas.

Pode-se destacar como limitação do presente estudo, o fato deste utilizar medidas duplamente indiretas (dobras cutâneas) para o estabelecimento da medida critério de gordura corporal, entretanto, no contexto da avaliação física em academias de ginástica, as medidas antropométricas são comumente utilizadas. Ainda pode-se destacar que os sujeitos do atual estudo foram selecionados por um procedimento não probabilístico em frequentadores de academia.

Os resultados aqui apresentados nos conduzem a concluir que existe uma relação positiva entre o \% GC e os indicadores antropométricos analisados, sendo que o IMC e a CA apresentaram maior força nas correlações para o sexo feminino e masculino, respectivamente. Além disso, mostram que a
RCE e CC são indicadores antropométricos que podem ser utilizados como alternativa da utilização da RCQ. Sugere-se que futuros estudos verifiquem se a combinação de indicadores antropométricos podem melhor explicar as variações no \% GC.

\section{REFERÊNCIAS}

Alvarez, M. M., Vieira, A. C. R., Sichieri, R., \& Veiga, G. V. (2008). Associação das medidas antropométricas de localização de gordura central com os componentes da síndrome metabólica em uma amostra probabilística de adolescentes de escolas públicas. Arquivos Brasileiros de Endocrinologia e Metabologia, 52 (4), 640-657.

Associação Brasileira para o Estudo da Obesidade e da Śndrome Metabólica - ABESO (1998). Consenso latino-americano em obesidade. Consultado em 5 de Maio de 2009, a partir de http://www.abeso.org.br/pdf/consenso.pdf

Callaway, A. R., Chumlea, W. C., Bouchard, C., Himes, J. H., Lohman, T. G., Martin, A. D., et al. (1991). Circunferences. In T. G. Lohman, A. F. Roche, \& R. Martorell (Eds.), Anthropometric standardization reference manual (pp. 39-54). Champaign, IL: Human Kinetics.

Carvalho, A. B. R., \& Pires Neto, C. S. (1999). Composição corporal através dos métodos de pesagem hidrostática e impedância bioelétrica em universitários. Revista Brasileira de Cineantropometria e Desempenho Humano, 1(1), 18-23.

Cavalcanti, C. B. S., Carvalho, S. C. B., \& De Barros, M. V. G. (2009). Indicadores antropométricos de obesidade abdominal: Revisão dos artigos indexados na biblioteca SciELO. Revista Brasileira de Cineantropometria e Desempenho Humano, 11 (2), 217-225.

Costa, R. F., Guiselini, M., \& Fisberg, M. (2007). Correlação entre porcentagem de gordura e índice de massa corporal de freqüentadores de academia de ginástica. Revista Brasileira de Ciência e Movimento, 15(4), 39-46.

Daniels, S. R., Khoury, P. R., \& Morrinson, J. A. (2000). Utility of different measures of body fat distribution in children and adolescents. American Journal of Epidemiology, 152, 1179-1184.

Ellis, K. J. (2000). Human body composition: In vivo methods. Physiology Review, 80, 649-680. 
Flegal, K. M., Shepherd, J. A., Looker, A. C., Graubard, B. I., Borrud, L. G., Ogden, C. L., et al. (2009). Comparisons of percentage body fat, body mass index, waist circumference, and waist-stature ratio in adults. American Journal of Clinical Nutrition, 80, 500-558.

Goodpaster, B. H., Krishnaswami, S., Harris, T. B., Katsiaras, A., Kritchevsky, S. B., Simonsick, E. M., et al. (2005). Obesity, regional body fat distribution, and the metabolic syndrome in older men and women. Archives of Internal Medicine, 165, 777-783.

Gordon, C. C., Chumlea, W. C., \& Roche, A. F. (1991). Stature, recumbent length, and weight. In T. G. Lohman, A. F. Roche, \& R. Martorell (Eds). Anthropometric standardization reference manual (pp. 3-8). Champaign, IL: Human Kinetics.

Harrison, G. G., Buskirk, E. R., Carter, J. E. L., Johston, F. E., Lohman, T. G., Pollock, M. L., et al. (1991). Skinfold thickness and measurement technique. In T. G. Lohman, A. F. Roche, \& R. Martorell (Eds.), Anthropometric standardization reference manual (pp. 55-80). Champaign, IL: Human Kinetics.

Hasselmann, M. H., Faerstein, E., Werneck, G. L., Chor, D., \& Lopes, C. S. (2008). Associação entre circunferência abdominal e hipertensão arterial em mulheres: Estudo Pró-Saúde. Caderno de Saúde Pública, 24(5), 1187-1191.

Ho, S. Y., Lam, T. H., \& Janus, E. D. (2003). Waist to stature ratio is more strongly associated with cardiovascular risk factors than other simple anthropometric indices. Annals of Epidemiology, 13(10), 683-691.

Huang, K. C., Lin, W. Y., Lee, L. T., Chen, C. Y., Lo, H., Hsia, H. H., et al. (2002). Four anthropometric indices and cardiovascular risk factors in Taiwan. International Journal of Obesity and Related Metabolic Disorders, 26(8), 10601068.

Hughes, V. A., Roubenoff, R., Wood, M., Frontera, W. R., Evans, W. J., \& Fiatarone, M. A. (2004). Singh anthropometric assessment of 10-y changes in body composition in the elderly. American Journal of Clinical Nutrition, 80, 475482.

Jackson, A. S., \& Pollock, M. L. (1978). Generalized equations for predicting body density of men. British Journal of Nutrition, 49, 497-504.

Jackson, A. S., Pollock, M. L., \& Ward, A. N. N. (1980). Generalized equations for predicting body density of women. Medicine and Science in Sports and Exercise, 12(3), 175-182.

Janssen, I., Heymsfield, S. B., Allison, D. B., Kotler, D. P., \& Ross, R. (2002). Body mass index and waist circumference independently contribute to the prediction of nonabdominal, abdominal, subcutaneous, and visceral fat. American Journal of Clinical Nutrition, 75, 683-688.

Lin, W. Y., Lee, L.T., Chen, C.Y., Lo, H. O., Hsia, H. H., Liu, I. L., et al. (2002). Optimal cut-off values for obesity: Using simple anthropometric indices to predict cardiovascular risk factors in Taiwan. International Journal of Obesity and Related Metabolic Disorders, 26(9), 1232-1238.

Mancini, M. (2001). Obstáculos diagnósticos e desafios terapêuticos no paciente obeso. Arquivos Brasileiros de Endocrinologia e Metabologia, 45, 584-608.

Matsudo, S. M., Matsudo, V. K. R., \& Barros Neto, T. L. (2000). Impacto do envelhecimento nas variáveis antropométricas, neuromotoras e metabólicas da aptidão física. Revista Brasileira de Ciência e Movimento, 8(4), 21-32.

National Heart Lung and Blood Institute - NHLBI (1998). Clinical guidelines on the identification, evaluation, and treatment of overweight and obesity in adults: Executive summary. American Journal of Clinical Nutrition, 68, 899-917.

Page, J. H., Rexrode, K. M., Hu, F., Albert, C. M., Chae, C. U., \& Manson, J. E. (2009). Waistheight ratio as a predictor of coronary heart disease among woman. Epidemiology, 20(3), 1-6.

Pereira, R. A., Sichieri, R., \& Marins, M. R. (1999). Razão cintura/quadril como preditor da hipertensão arterial. Caderno de Saúde Pública, 15(2), 333-344.

Pitanga, F. J. G., \& Lessa, I. (2006). Razão cinturaestatura como discriminador do risco coronariano de adultos. Revista da Associação Médica Brasileira, 52(3), 157-161.

Rech, C. R., Petroski, E. L., Silva, R. C. R., \& Silva, J. C. N. (2006). Indicadores antropométricos de excesso de gordura corporal em mulheres. Revista Brasileira de Medicina do Esporte, 12(3), 119-124.

Rexrode, R. M., Carey, V. J., Hennekens, C. H., Walters, E. E., Colditz, G. A., Stampfer, M. J., et al. (1998). Abdominal adiposity and coronary heart disease in women. The Journal of the American Medical Association, 280, 1843-1848. 
Ribeiro, A. B., Gimeno, S. G. A., Andreoni, S., \& Ferreira, S. R. G. (2006). Japanese Brazilian diabetes group: Should body mass index be adjusted for relative sitting height in crosssectional studies of chronic diseases in Japanese-Brazilians? Cadernos de Saúde Pública, 22, 1691-1697.

Silva, J. L. T., Barbosa, D. S., Oliveira, J. A., \& Guedes, D. P. (2006). Distribuição centrípeta da gordura corporal, sobrepeso e aptidão cardiorrespiratória: Associação com sensibilidade insulínica e alterações metabólicas. Arquivos Brasileiros de Endocrinologia E Metabologia, 50 (6), 1034-1040.

Siri, W. E. (1961). Body composition from fluid spaces and density: Analysis of methods. In J. Brozek \& A. Henschel (Eds.), Techniques for measuring body composition (pp. 223-244). Washington, DC: National Academic of Science.

Wang, J., Thornton, J. C., Bari, S., Williamson, B., Gallagher, D., Heymsfield, S. B., et al. (2003). Comparisons of waist circumferences measured at 4 sites. The American Journal of Clinical Nutrition, 77(2), 379-382.

Wannamethee, S. G., Shaper, A. G., Lennon, L., \& Whincup, P. H. (2007). Decreased muscle mass and increased central adiposity are independently related to mortality in older men. The American Journal of Clinical Nutrition, 86 (5), 1339-1346.

World Health Organization - WHO. (1995). Physical status: the use and interpretation of anthropometry. Geneve: Autor. 